\title{
The corona of HD 199178 (V 1794 Cygni)
}

\author{
P. Gondoin ${ }^{\star}$
}

\author{
European Space Agency, ESTEC - Postbus 299, 2200 AG Noordwijk, The Netherlands
}

Received 20 May 2003 / Accepted 1 October 2003

\begin{abstract}
HD 199178 (V 1794 Cygni), a chromospherically active late-type giant with a high X-ray luminosity, was observed by the XMM-Newton space observatory. Series of lines of highly ionized Fe and several Lyman lines of hydrogen-like ions are visible in the reflection grating spectra, most notably from $\mathrm{O}$ and $\mathrm{Ne}$. Analysis results suggest a scenario where the corona of HD 199178 is dominated by large magnetic structures similar in size to interconnecting loops between solar active regions but significantly hotter. The surface area coverage of these active regions may approach up to a $90 \%$. An hypothesis is that the interaction of these structures themselves induces a flaring activity in a small scale not visible in the EPIC light curves that is responsible for heating HD 199178 plasma to coronal temperatures of $T \geq 10^{7} \mathrm{~K}$. The intense X-ray activity of HD 199178 is related to its evolutionary position at the bottom of the red giant branch. It is anticipated that its rotation will spin-down in the future with the effect of decreasing its helicity related, dynamo driven activity and suppressing large scale magnetic structures in its corona.
\end{abstract}

Key words. stars: individual: HD 199178 - stars: activity - stars: coronae - stars: evolution - stars: late-type - stars: x-ray

\section{Introduction}

HD 199178 (V 1794 Cygni), an active late-type giant, was classified as an FK Comae type star by Bopp \& Stencel (1981). HD 199178 is an extremely fast rotating star with a photometric rotation period of $3.337 \pm 0.001$ days (Bopp et al. 1983; Jetsu et al. 1990) and $v \sin i$ included between $65.4 \mathrm{~km} \mathrm{~s}^{-1}$ (Fekel 1997) and $74 \mathrm{~km} \mathrm{~s}^{-1}$ (Dempsey et al. 1992). Its spectral type was initially determined by Herbig (1958) to be G5 IIIIV. Huenemoerder (1986) then suggested a classification of G7 III-IV from its optical spectrum.

Because of its rapid rotation, HD 199178 is well suited for surface mapping using Doppler imaging techniques (Hackmann et al. 2001) that provide detailed information on the photospheric temperature distribution. Vogt (1988) presented a first Doppler image of HD 199178 that shows a big cool polar cap and one equatorial spot. Maps constructed by Strassmeier et al. (1999) show the same morphology but with several equatorial spots suggestive of a high level of magnetic activity in the photosphere of the star. Important solar-type activity on HD 199178 is also supported by VLBI observations (Lestrade et al. 1999) of a large outburst of $300 \mathrm{mJy}$ on 1994 May 14 with evidence of fast motion of the radio emitting region which is reminiscent of coronal mass ejection on the Sun. Other indicators of strong magnetic activity in the atmosphere of HD 199178 include an X-ray luminosity (Hünsch et al. 1998) exceptionally high for a red giant, similar to that of an FK Comae type star (Gondoin 1999). I report on analysis results

\footnotetext{
* e-mail: pgondoin@rssd.esa.int
}

of X-ray spectra of HD 199178 registered during two observations performed in November 2002 by the XMM-Newton observatory. The observations were conducted with the aim to improve our understanding of the magnetic activity on giants like HD 199178 by investigating the origin of its high X-ray luminosity and the structure of its X-ray corona.

This paper is organized as follows. Section 2 provides the stellar parameters of HD 199178 and compares the evolution status of this star with those of nearby single field giants in light of Hipparcos parallaxes (ESA 1997). Section 3 describes the X-ray observations of HD 199178 and the data reduction procedures. Section 3 then presents the integrated flux measurements and their temporal behaviour during the observations. Section 4 describes the spectral analysis of the EPIC and RGS datasets. Finally, a physical interpretation of the analysis results is proposed in Sect. 5. In this last section, the structure of HD 199178 corona and its possible evolution is discussed within the frame of stellar activity evolution across the Hertzsprung gap.

\section{HD 199178 stellar parameters}

The absolute magnitude of HD 199178 was calculated from its $V$ magnitude, visual extinction and Hipparcos parallax (ESA 1997). The brightest $V$ magnitude ( $V=7.05$ in 1986.84; Jetsu et al. 1999) of HD 199178 observed between 1975 and 1996 was adopted as its least spotted magnitude. The Hipparcos spacecraft measured a trigonometric parallax of 10.68 mas corresponding to a distance of $94 \pm 6 \mathrm{pc}$. The visual extinction of the star was calculated by applying Chen et al. (1998) model with the upper distance limit derived from the Hipparcos catalogue. The visual extinction is smaller than the magnitude 

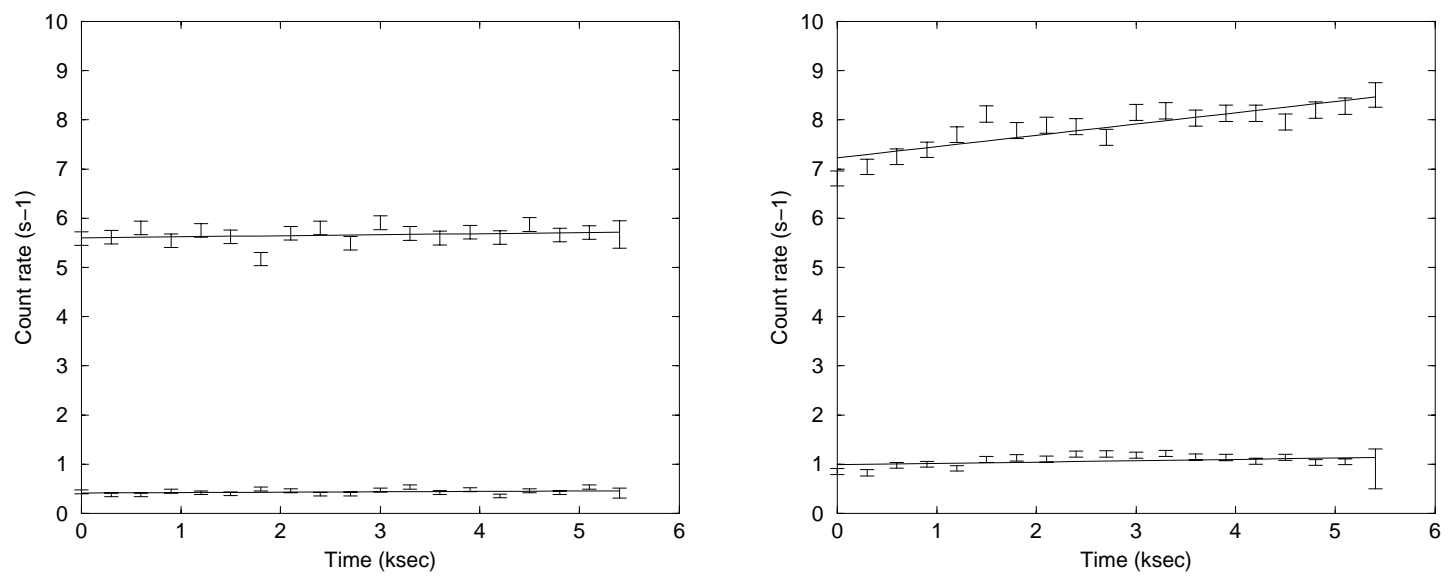

Fig. 1. Light curves of HD 199178 during revolutions 536 (left) and 542 (right) obtained with the EPIC p-n camera. In each graph, the upper curve is the count rate within the 0.3 to $2 \mathrm{keV}$ band and the lower curve is the count rate within the 2 to $10 \mathrm{keV}$ band. The events are binned in $300 \mathrm{~s}$ time intervals and the background contribution has been subtracted.

Table 1. Top: $V$ magnitudes, parallaxes, extinctions and absolute magnitudes of HD 199178. The brightest $V$ magnitude of the least-spotted photosphere is quoted. Middle: spectral type, color indices and effective temperature. The temperature of the quiescent photosphere outside starspots is given as derived from Doppler imaging techniques. Bottom: estimated stellar parameters of HD 199178.

\begin{tabular}{cccc}
\hline \hline$V$ & par (mas) & $A_{V}$ & $M_{V}$ \\
7.05 & $10.68 \pm 0.73$ & 0.05 & $2.16-2.32$ \\
\hline Sp. Type & $B-V$ & $V-I$ & $T_{\text {eff }}(\mathrm{K})$ \\
G5 III & 0.785 & 0.82 & 5450 \\
\hline$L\left(L_{\odot}\right)$ & $R\left(R_{\odot}\right)$ & $v_{\text {eq }}\left(\mathrm{km} \mathrm{s}^{-1}\right)$ & $M\left(M_{\odot}\right)$ \\
$12-15$ & $4.6-5.5$ & $70-83$ & $1.7-1.9$ \\
\hline
\end{tabular}

variation due to rotational modulation by photospheric spots. The stellar luminosity was then derived using the bolometric correction vs effective temperature data of Flower (1996).

Jetsu et al. (1990) estimated a $T_{\text {eff }}$ value for HD 199178 in the range $5300 \mathrm{~K}$ to $5450 \mathrm{~K}$. Strassmeier et al. (1999) found the best fits for photospheric line profiles and $B V$ photometry when using a nominal photospheric temperature in the range 5400-5500 K. Following these authors, we adopted $T_{\text {eff }}=5450 \mathrm{~K}$ as the default temperature of the unspotted photosphere that is the most consistent with the color indices and the G5 III-IV spectral classification. The mass of HD 199178 is estimated to $1.7-1.9 M_{\odot}$ from its position with respect to theoretical evolutionary tracks inferred from grids of stellar models with a near solar metallicity $(Z=0.02)$ provided by Schaller et al. (1992). The models use opacities provided by Rogers \& Iglesias (1991) and Kurucz (1991) and their convection parameters (i.e. mixing length ratio and overshooting parameter) have been calibrated using the red giant branch of a wide range of clusters. The star most likely originates from an early A type, single star as it evolves in the giant domain.

The radius of HD 199178 was calculated from its luminosity and effective temperature. The photometric period $(P=$ $3.337 \pm 0.01$ days; Bopp et al. 1983; Jetsu et al. 1990) and the radius of the star were then used to estimate its equatorial velocity (see Table 1). Comparison with the projected rotational velocity $\left(v \sin i=71.5 \pm 1.0 \mathrm{~km} \mathrm{~s}^{-1}\right.$; Strassmeier et al. 1999) derived spectroscopically indicates a large inclination angle $\left(i>58^{\circ}\right)$ of the star's polar axis onto the line of sight. This effect contributes to explain the large rotational velocity of HD 199178 that is consistent with the hypothesis that the star is a normal $\mathrm{G}$ giant that has evolved from a single A type progenitor.

\section{Observations and data reduction}

HD 199178 was observed twice by the XMM-Newton space observatory (Jansen et al. 2001), in revolutions 536 on 2002 November 11, and 542 on 2002 November 23 (see Table 2). The observations were conducted with the EPIC cameras (Strüder et al. 2001; Turner et al. 2001) operating in full frame mode (Ehle et al. 2001). Spectra were recorded simultaneously with the RGS reflection grating spectrometers (den Herder et al. 2001). Processing of the raw event dataset was performed using the "emchain", "epchain" and "rgsproc" pipeline tasks of the XMM-Newton Science Analysis System (SAS version 5.3.0). The large count rate of the target produced pile-up effects in the core of the telescope point spread functions registered by the EPIC cameras. In order to reject these ambiguous events, the source spectra in the EPIC cameras were built from photons detected within an annulus of radius included between $4.5^{\prime \prime}$ and $25^{\prime \prime}$ from the target boresight. The spectra and lightcurves of HD 199178 were extracted and analyzed following a standard procedure (Gondoin 2002, 2003a).

Figure 1 shows the light curves of HD 199178 obtained during revolution 536 and 542 with the $\mathrm{p}-\mathrm{n}$ camera after subtraction of background events. In the 0.3 to $2 \mathrm{keV}$ energy band, the count rate was about $40 \%$ higher in revolution 542 with an average count rate of $7.85 \pm 0.45 \mathrm{~s}^{-1}$ compared with a count rate of $5.66 \pm 0.16 \mathrm{~s}^{-1}$ in revolution 536 . In the 2 to $10 \mathrm{keV}$ energy band, the count rate was more than a factor of two higher in revolution 542 with an average count rate of $1.06 \pm 0.11 \mathrm{~s}^{-1}$ compared with a count rate of $0.44 \pm 0.05 \mathrm{~s}^{-1}$ in revolution 536 . The $0.3-2 \mathrm{keV}$ over $2-10 \mathrm{keV}$ count rate ratios were $13 \pm 2$ and $7.3 \pm 1.5$ during revolutions 536 and 542 respectively. The count rate in the low energy band only slightly increased by $\approx 2 \%$ over 
Table 2. HD 199178 observation log during XMM-Newton revolutions 536 and 542.

\begin{tabular}{cccccc}
\hline \hline Rev. & Experiment & Filter & Mode & Start Exp. (UT) & Exp. Duration \\
\hline \multirow{4}{*}{536} & MOS1 & Medium & Prime Full & 2002-11-11@23:49:53 & $7167 \mathrm{~s}$ \\
& MOS2 & Medium & Prime Full & 2002-11-11@23:49:53 & $7167 \mathrm{~s}$ \\
& p-n & Medium & Prime Full & 2002-11-12@00:12:24 & $5546 \mathrm{~s}$ \\
& RGS1 & & Spec + Q & 2002-11-11@23:48:48 & $7385 \mathrm{~s}$ \\
& RGS2 & & Spec + Q & 2002-11-11@23:48:48 & $7385 \mathrm{~s}$ \\
\hline \multirow{5}{*}{542} & MOS1 & Medium & Prime Full & 2002-11-23@23:03:45 & $7167 \mathrm{~s}$ \\
& MOS2 & Medium & Prime Full & 2002-11-23@23:03:45 & $7167 \mathrm{~s}$ \\
& p-n & Medium & Prime Full & 2002-11-23@23:26:16 & $5546 \mathrm{~s}$ \\
& RGS1 & & Spec + Q & 2002-11-23@23:02:40 & $7385 \mathrm{~s}$ \\
& RGS2 & & Spec + Q & 2002-11-23@23:02:40 & $7385 \mathrm{~s}$ \\
\hline
\end{tabular}

Table 3. X-ray luminosities (corrected for interstellar absorption) of HD 199178 in the $0.3-2 \mathrm{keV}$ and 2-10 keV measured with the combined EPIC MOS and pn cameras. The percentage contribution in luminosity of hot plasmas $(k T>1 \mathrm{keV})$ is indicated between bracketts. The hardness ratio (hr) is defined as $h r=\left(L_{2-10 \mathrm{keV}}-\right.$ $\left.L_{0.3-2 \mathrm{keV}}\right) /\left(L_{2-10 \mathrm{keV}}+L_{0.3-2 \mathrm{keV}}\right)$.

\begin{tabular}{cccc}
\hline \hline Obs. & $\begin{array}{c}L_{0.3-2 \mathrm{keV}} \\
\left(10^{30} \mathrm{erg} \mathrm{s}^{-1}\right)\end{array}$ & $\begin{array}{c}L_{2-10 \mathrm{keV}} \\
\left(10^{30} \mathrm{erg} \mathrm{s}^{-1}\right)\end{array}$ & $h r$ \\
\hline Rev. 536 & 14.5 & 5.4 & -0.46 \\
& $(75 \%)$ & $(97 \%)$ & \\
Rev. 542 & 21.1 & 14.3 & -0.19 \\
& $(85 \%)$ & $(99 \%)$ & \\
\hline
\end{tabular}

$5.6 \mathrm{ksec}$ in revolution 536 . It increased by about $20 \%$ during revolution 542 over the same duration.

The spectral analyses of each observation were conducted separately. Spectral fitting of the EPIC data (see Sect. 4.1) during these two periods yields flux measurements in the $0.3-2 \mathrm{keV}$ and 2-10 keV bands. These measurements were converted into X-ray luminosities $L_{0.3-2 \mathrm{keV}}$ and $L_{>2} \mathrm{keV}$ using Hipparcos parallax $(\pi=10.68$ mas; ESA 1997). The luminosities are given in Table 3 which also provides the hardness ratio $h r$ of the X-ray emission defined as $h r=\left(L_{2-10 \mathrm{keV}}-\right.$ $\left.L_{0.3-2 \mathrm{keV}}\right) /\left(L_{2-10 \mathrm{keV}}+L_{0.3-2 \mathrm{keV}}\right)$. Table 3 shows that the X-ray spectrum of HD 199178 is harder in revolution 542. Compared with revolution 536, the X-ray luminosity of HD 199178 during revolution 542 was $46 \%$ higher in the $0.3-2 \mathrm{keV}$ band and a factor of 2.6 larger in the high energy band.

\section{Spectral analysis}

\subsection{Analysis of EPIC data}

The two EPIC datasets (see Fig. 2) were fitted separately with the MEKAL optically thin plasma emission model (Mewe et al. 1985). The spectral fitting was performed in the $0.3-10 \mathrm{keV}$ spectral bands for both revolutions. The interstellar hydrogen column density was left free to vary. $N_{\mathrm{H}}$ values in the range $(2.6-3.0) \times 10^{20} \mathrm{~cm}^{-2}$ were derived from the analysis of the two datasets. This range measures the H I column density up to HD 199178 and is expected to be lower than the total galactic
$\mathrm{H}$ I column density $N_{\mathrm{H}}=1.17 \times 10^{22} \mathrm{~cm}^{-2}$ (Dickey \& Lockman 1990) in this direction which also includes the H I column density contribution beyond HD 199178. No single temperature plasma model that assumes either solar photospheric (Anders \& Grevesse 1989) or non solar abundances can fit the data, as unacceptably large values of $\chi^{2}$ were obtained. The MEKAL plasma models with two components at different temperatures prove barely acceptable with a $\chi^{2} \approx 1.3$ for the two datasets. Also the large variation of the best fit parameters between the two revolution datasets looks unrealistic. The coronal emission measure distribution has been proposed to be double peaked for many stars (Schrijver et al. 1995; Mewe et al. 1996; Güdel et al. 1997a, 1997b). However, as spatially unresolved observations gain in spectral resolution and signal to noise ratio, the amount of details in the spectra of stellar coronae which must be reproduced increases reflecting the true complexity of the sources plasma. Multi-temperature models are now necessary to explain high-resolution spectra of stellar coronae (Dupree et al. 1993; Griffiths \& Jordan 1998; Bowyer et al. 2000). Hence, the EPIC spectra of HD 199178 were fitted using a MEKAL model with three components at different temperatures and with the same metallicity. The improvement in $\chi^{2}$ statistics compared with the two temperatures model $\left(\Delta \chi^{2} / \Delta v=40\right.$ for 698 degrees of freedom and $\Delta \chi^{2} / \Delta v=71.4$ for 972 degrees of freedoms on the best fit to revolution 536 and 542 datasets, respectively) is significant to a $>99 \%$ confidence level using the F-statistic. The addition of a fourth component to the model does not improve the quality of the spectral fit.

The temperatures of the different plasma components remain similar for revolution 536 and 542. The average element abundance in HD 199178 corona is found to be lower than the solar photospheric value (see Table 4). No significant variations of abundance are detected between the two revolutions. The three component model suggests that most of the emission measure of the "hot" plasma is located around $T=1.6 \times 10^{7} \mathrm{~K}$ in revolution 536 and 542 but has a large contribution above $T=6 \times 10^{7} \mathrm{~K}$ in revolution 542 . The temperature of this very hot plasma component is not well constrained. Hot $\left(T>1.5 \times 10^{7} \mathrm{~K}\right)$ plasma on HD 199178 is the main source of X-ray emission both in the soft and in the hard $\mathrm{X}$-ray band. It contributes to more than $97 \%$ of the X-ray luminosity above $2 \mathrm{keV}$. Table 4 shows that the higher X-ray 

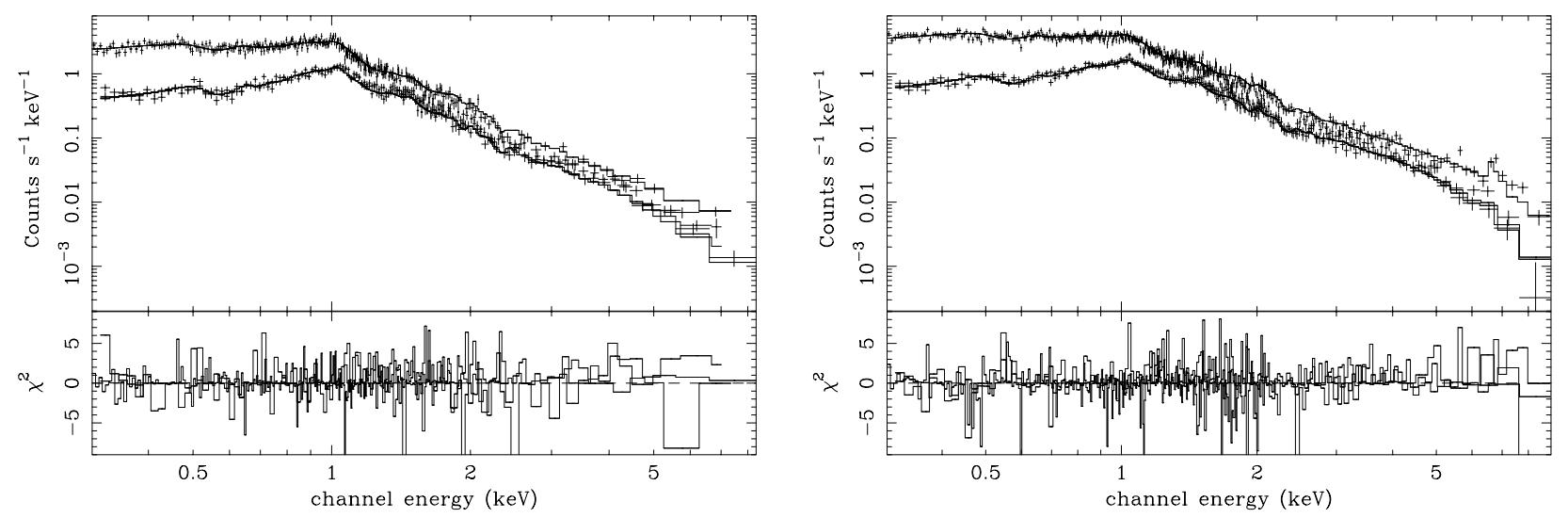

Fig. 2. Best fit model to revolution 536 (left) and revolution 542 (right) EPIC spectra. The data (crosses) and spectral fit (solid line) to the EPIC pn (upper curve) and EPIC MOS (lower curve) spectra are shown in the upper panel. The $\chi^{2}$ contributions are given in the lower panel of each graph.

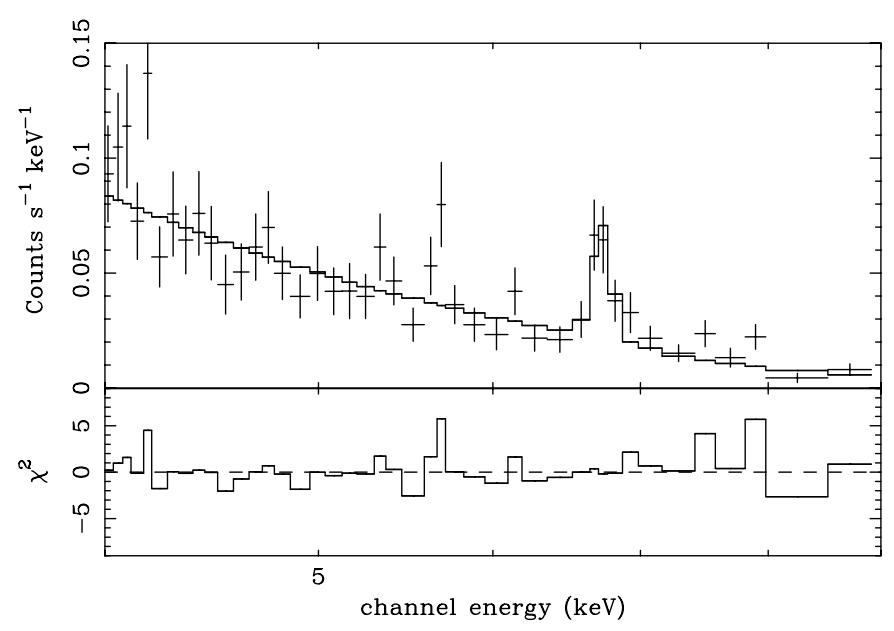

Fig. 3. Comparison of HD 199178 spectrum obtained during revolution 542 with a best fit power law model + gaussian model in the $3-10 \mathrm{keV}$ range.

luminosity of HD 199178 in revolution 542 both in the soft and in the hard energy band is due to a larger emission measure of this hot plasma.

One major feature of HD 199178 spectrum during revolution 542 is the presence of a high energy tail and of an emission feature around $7 \mathrm{keV}$ attributed to an iron $\mathrm{K}$ emission line (see Fig. 3). This component is not detected in revolution 536 data. The iron $\mathrm{K} \alpha$ fluorescent line energy is an increasing function of the ionization state. It rises slowly from $6.40 \mathrm{keV}$ in Fe I to $6.45 \mathrm{keV}$ in Fe XVII (neon-like) and then increases steeply with the escalating number of vacancies in the L-shell to $6.7 \mathrm{keV}$ in Fe XXV and $6.9 \mathrm{keV}$ in Fe XXVI (House 1969; Makishima 1986). Spectral fits to the EPIC $\mathrm{p}-\mathrm{n}$ spectra above $4 \mathrm{keV}$ by a powerlaw and a Gaussian line model give a power law with a slope $\Gamma=2.4 \pm 0.1$ and an $\mathrm{Fe} \mathrm{K}$ line at $6.71 \pm 0.02 \mathrm{keV}$ with an equivalent width $E W \approx 400 \pm 20 \mathrm{eV}$. The improvement of the power law fit with an additional Gaussian line is significant $\left(\Delta \chi^{2} / \Delta v=10\right.$ for 81 degrees of freedom) to a $>99 \%$ confidence level using the F-statistic. This result indicates the presence of iron in high Fe XXVI states of ionization during revolution 542.
For a collisionally dominated optically thin coronal plasma, the Fe XXVI ion concentration reaches a maximum value in the $(2-10) \times 10^{7} \mathrm{~K}$ temperature range (Raymond-Smith 1977), in agreement with the temperature of the hot plasma component derived by spectral fitting. This supports the thermal origin of the Fe K emission.

Among all of the different changes, eruption and instabilities which are seen on the Sun, the ones which are labeled "flares" all have in common material heated to temperatures of $10^{7} \mathrm{~K}$ or higher (Golub \& Pasachoff 1997; Reale et al. 2001). Such temperatures are not seen in the non-flaring corona, and events which do not produce such hot plasma do not seem to be called flares. The emission measure of some active stellar coronae has two peaks, one at at a few $10^{6} \mathrm{~K}$ and the other around $10^{7} \mathrm{~K}$, and it has been proposed that the higher temperature peak is due to continuous flaring activity (Drake et al. 2000; Sanz-Forcada et al. 2002). However, such a flaring activity is expected to induce count rate fluctuations that are not observed in the EPIC light curves of HD 199178. Hence, although suggestive, the existence of significant amounts of $>10^{7} \mathrm{~K}$ material and the detection of Fe XXVI emission cannot be regarded as a definite proof for the presence of flares in the corona of HD 199178.

\subsection{Analysis of RGS data}

Figure 4 shows the RGS spectra of HD 199178 averaged over revolution 536 and 542. Each spectrum is the sum of the two spectra simultaneously obtained with the RGS1 and RGS2 reflection grating spectrometers on board XMM-Newton. Line fluxes and positions were measured using the XSPEC package by fitting simultaneously the RGS1 and RGS2 spectra with a sum of narrow Gaussian emission lines convolved with the response matrices of the RGS instruments. The continuum emission was described using Bremstrahlung models at the temperatures of the hot plasma components inferred from the analysis of EPIC data. For line identification, we required only that the wavelength coincidence be comparable to the spectral resolution of the RGS spectrometers, namely $0.04 \AA$ over the 5 to $35 \AA$ wavelength range. In the X-ray domain, several 
Table 4. Best fit parameters to EPIC data using a 3 components MEKAL model (Mewe et al. 1985) with variable abundance and a variable hydrogen column density.

\begin{tabular}{ccc}
\hline \hline & Rev. 536 & Rev. 542 \\
\hline$Z$ & $0.20 \pm 0.02$ & $0.23 \pm 0.03$ \\
$k T_{1}(\mathrm{keV})$ & $0.70 \pm 0.02$ & $0.68 \pm 0.03$ \\
$E M_{1}\left(10^{52} \mathrm{~cm}^{-3}\right)$ & $43 \pm 7$ & $35 \pm 6$ \\
$k T_{2}(\mathrm{keV})$ & $1.33 \pm 0.05$ & $1.44 \pm 0.06$ \\
$E M_{2}\left(10^{52} \mathrm{~cm}^{-3}\right)$ & $126 \pm 12$ & $143 \pm 15$ \\
$k T_{3}(\mathrm{keV})$ & $7.3 \pm 3.1$ & $6.5 \pm 1.0$ \\
$E M_{3}\left(10^{52} \mathrm{~cm}^{-3}\right)$ & $29 \pm 12$ & $111 \pm 12$ \\
$\chi^{2}$ & $829 / 698$ d.o.f. $=1.19$ & $1111 / 972$ d.o.f. $=1.14$ \\
\hline
\end{tabular}

Table 5. Measured fluxes of the strongest lines in the RGS spectra of HD 199178 obtained during revolutions 536 and 542. Predicted line positions, line identifications and temperatures of maximum line formation are given.

\begin{tabular}{cccccccc}
\hline \hline $\begin{array}{c}\lambda_{\text {pred }} \\
(\AA)\end{array}$ & $\begin{array}{c}\lambda_{\text {rev 536 }} \\
(\AA)\end{array}$ & $\begin{array}{c}\lambda_{\text {rev 542 }} \\
(\AA)\end{array}$ & Ion & line ID & $\begin{array}{c}\log \left(T_{\mathrm{m}}\right) \\
\log (\mathrm{K})\end{array}$ & $\begin{array}{c}F_{\text {rev 536 }} \\
\left(10^{-6} \mathrm{~cm}^{-2} \mathrm{~s}^{-1}\right)\end{array}$ & $\begin{array}{c}F_{\text {rev 542 }} \\
\left(10^{-6} \mathrm{~cm}^{-2} \mathrm{~s}^{-1}\right)\end{array}$ \\
\hline 12.13 & 12.16 & 12.16 & Ne X & H1AB & 6.80 & $330 \pm 80$ & $380 \pm 80$ \\
12.12 & & & Fe XVII & $4 \mathrm{C}$ & 6.75 & & \\
12.91 & 12.92 & \multirow{2}{*}{12.92} & Fe XX & & 7.00 & $<130$ & $170 \pm 70$ \\
12.93 & & & Ni XX & & 6.90 & & \\
14.07 & 14.09 & 14.09 & Ni XIX & Ne8AB & 6.70 & $160 \pm 50$ & $110 \pm 50$ \\
14.96 & 14.94 & 14.94 & Fe XIX & & 6.90 & $120 \pm 40$ & $95 \pm 50$ \\
16.08 & 16.10 & 16.10 & Fe XVIII & F3 & 6.80 & $100 \pm 50$ & $100 \pm 50$ \\
16.11 & & & Fe XIX & O2 & 6.90 & & \\
17.10 & 17.22 & 17.22 & Fe XVII & M2 & 6.70 & $<70$ & $70 \pm 50$ \\
18.97 & 19.08 & 19.08 & O VIII & H1AB & 6.50 & $115 \pm 50$ & $180 \pm 60$ \\
\hline
\end{tabular}

candidate lines may exist within this acceptable wavelength coincidence range. Hence, we only looked for resonance transitions of abundant elements and predicted line intensities using spectra of the Sun (Doschek \& Cowan 1984) and of Capella (Brinkman et al. 2000). Series of lines of highly ionized Fe and several lines of the Ly series are visible in RGS spectra, most notably from $\mathrm{O}$ and $\mathrm{Ne}$. Table 5 lists the measurements of lines that are statistically significant. Line fluxes corrected for interstellar absorption $\left(N_{\mathrm{H}}=2.8 \times 10^{20} \mathrm{~cm}^{-2}\right)$ on the line of sight to HD 199178 are reported. Their temperatures of maximum formation range between $3 \times 10^{6} \mathrm{~K}$ and $10^{7} \mathrm{~K}$ suggesting that the corresponding ions are mainly associated with the plasma component at $0.7 \mathrm{keV}$ inferred from EPIC data. Lines such as the $\mathrm{O}$ VIII and Ne X lines have emissivity functions quite spread in temperature. The flux measurement of some lines such as $\mathrm{Ne} X$ $(12.13 \AA)$ is affected by blends. This could explain that its flux (see Table 5) is high compared with the value expected from the analysis of EPIC spectra. No significant variations of line intensities are observed between revolutions 536 and 542.

The low energy RGS spectra were fitted with a VMEKAL model with three components at different temperatures. The VMEKAL model generates a spectrum of hot diffuse gas with line emission from several elements based on the calculation of Mewe et al. (1985) with Fe L calculations by Liedahl (1995). Hence, three electron temperatures and electron densities are assumed for the entire ensemble of element charge states and in particular for iron, oxygen and neon which produce the most prominent lines. This assumption turns out to be fairly adequate within the observational uncertainties of the present spectrum (see Fig. 5). The fit was performed in the spectral range from $6 \AA$ to $40 \AA$. The model temperatures of the cool, midtemperature and hot plasma components were frozen to the values derived from EPIC data (see Table 4) since their determination requires an accurate measurement of the X-ray continuum which cannot be reliably measured from the RGS spectra (see Fig. 4) due to a moderate spectral resolution and signal to noise ratio. The abundances of the $\mathrm{O}$ and $\mathrm{Ne}$ elements which give prominent lines in the considered spectral range were first tied to the abundance of the other elements (MODEL A). They were then allowed to vary independently but with the same value for all temperature components (MODEL B). Fitting results are given in Table 6 for revolution 536 (upper table) and revolution 542 (lower table). The fit supports the three components plasma model for the interpretation of the EPIC and RGS data. When left free to vary, the oxygen abundance $(0.25 \pm 0.04)$ is slightly higher than the average abundance $(0.12 \pm 0.04)$ of the other elements and the neon abundance is more than three times higher than the oxygen abundance. The improvement in $\chi^{2}$ fit statistics $\left(\Delta \chi^{2} / \Delta v=30\right.$ for 447 degrees of freedom and $\Delta \chi^{2} / \Delta v=33$ for 756 degrees of freedom, respectively for revolution 536 and 542) induced with variable $\mathrm{O}$ and $\mathrm{Ne}$ abundance is significant at $>99 \%$ confidence using the F-statistic. 
Table 6. Best fit parameters to RGS spectra in the $0.3-2.1 \mathrm{keV}$ range recorded in revolutions 536 (upper table) and 542 (lower table) using a three components VMEKAL model. The temperature of each component were frozen to the value derived from the analysis of EPIC data (see Table 4). The metallicity was left free to vary. The oxygen and neon abundances were first tied to the abundance of the other elements (MODEL A). They were then left free to vary independently but with the same value for the different temperature components (MODEL B).

\begin{tabular}{cccc}
\hline \hline Rev. 536 & Parameter & MODEL A & MODEL B \\
\hline & $k T_{1}(\mathrm{keV})$ & 0.70 & 0.70 \\
& $E M_{1}\left(10^{52} \mathrm{~cm}^{-3}\right)$ & $35 \pm 10$ & $25 \pm 7$ \\
VMEKAL & $k T_{2}(\mathrm{keV})$ & 1.33 & 1.33 \\
& $E M_{2}\left(10^{52} \mathrm{~cm}^{-3}\right)$ & $186 \pm 22$ & $173 \pm 21$ \\
(3 components) & $k T_{3}(\mathrm{keV})$ & 7.3 & 7.3 \\
& $E M_{3}\left(10^{52} \mathrm{~cm}^{-3}\right)$ & $93 \pm 25$ & $89 \pm 25$ \\
& $\mathrm{O}$ & 0.11 & $0.23 \pm 0.04$ \\
& $\mathrm{Ne}$ & 0.11 & $1.0 \pm 0.2$ \\
& Other abundances & $0.11 \pm 0.04$ & $0.11 \pm 0.03$ \\
& $\chi^{2}$ & $1.21(667 / 549$ d.o.f. $)$ & $1.11(608 / 547$ d.o.f. $)$ \\
\hline \hline Rev. 542 & Parameter & MODEL A & MODEL B \\
\hline & $k T_{1}(\mathrm{keV})$ & 0.68 & 0.68 \\
& $E M_{1}\left(10^{52} \mathrm{~cm}^{-3}\right)$ & $28 \pm 11$ & $19 \pm 8$ \\
& $k T_{2}(\mathrm{keV})$ & 1.44 & 1.44 \\
& $E M_{2}\left(10^{52} \mathrm{~cm}^{-3}\right)$ & $218 \pm 27$ & $207 \pm 27$ \\
& $k T_{3}(\mathrm{keV})$ & 6.5 & 6.5 \\
(3 components) & $E M_{3}\left(10^{52} \mathrm{~cm}^{-3}\right)$ & $35 \pm 10$ & $186 \pm 27$ \\
& $\mathrm{O}$ & 0.12 & $0.25 \pm 0.04$ \\
& $\mathrm{Ne}$ & 0.12 & $0.88 \pm 0.14$ \\
& Other abundances & $0.12 \pm 0.04$ & $0.11 \pm 0.01$ \\
& $\chi^{2}$ & $1.16(882 / 758$ d.o.f. $)$ & $1.08(816 / 756$ d.o.f. $)$ \\
\hline
\end{tabular}

It is worth noting that the emission measures derived by fitting the same model to EPIC and RGS spectra give consistent values for the low temperatures component (see Tables 4 and 6 model A) which mainly contribute to the X-ray emission though intense emission lines in the $0.3-2.1 \mathrm{keV}$ spectral range of RGS. The emission measures of the other components gives values sometimes different since the low ratio signal to noise and the moderate spectral resolution of the RGS spectra provide poor constraints to continuum emission through which hot $(>1.3 \mathrm{keV})$ plasmas mainly contribute.

Recent analysis of XMM-Newton and Chandra X-ray spectra find that a continuous emission measure distribution fits the spectra better and is more realistic physically (Audard et al. 2001a,b; Güdel et al. 2001b; Mewe et al. 2001). We tried to fit the RGS spectra of HD 199178 first using a plasma model with a simple continuous emission measure distribution which is a power law function of the temperature of the type $E M(T) \approx\left(T / T_{\max }\right)^{\alpha}$, where the maximum temperature of the plasma $T_{\max }$ and the slope of the emission $\alpha$ are treated as free parameters (Schmitt et al. 1990). The abundance of oxygen and neon were initially tied to the abundance of the other element. They were then allowed to vary independently. An acceptable fit to the RGS spectra (see Table 7) is obtained for both revolutions. The slope $(\alpha=1.3 \pm 0.2)$ indicates that the emission measure increase with temperature up to high temperatures $\left(k T_{\max }>2 \mathrm{keV}\right.$. The abundance of oxygen $(0.26 \pm 0.07)$ equals that of other elements and is identical to the value derived from the VMEKAL models (see Table 6). The fitting with a power law emission measure distribution also indicates a neon abundance enhancement (by about a factor of 3) relative to oxygen. We tried to fit the RGS spectra of HD 199178 with a more complex plasma model using a continuous emission measure distribution parameterized by the exponential of a sum of terms of a 6th order Chebyshev polynomial in the $\log (E M)-\log (T)$ plane (Lemen et al. 1989). The polynomial coefficients could not be constrained by the data due to the moderate signal to noise ratio.

The best fit models with either discrete or continuous emission measure distribution give consistent values of the oxygen and neon abundances. Using the Anders \& Grevesse (1989) table, the oxygen abundance (see Tables 6 and 7) translates into $\log (\epsilon(O))=8.33 \pm 0.12$, a value slightly lower than a recent measurement of the oxygen abundance in the solar photosphere $(\log (\epsilon(O))=8.69 \pm 0.05$; Allende Prieto \& Lambert 2001) which is itself smaller than older values usually cited. The measurement of abundances relative to hydrogen requires an accurate determination of the X-ray continuum which is modelled from the flux left over when all of the known emission lines in the VMEKAL model are included. Since no plasma spectroscopy code includes all of the emission lines, the missing weak emission lines could be misinterpreted as continuum flux (Schmitt et al. 1996), thereby raising the hydrogen abundance derived from the free-free continuum and lowering all of the metal abundances relative to hydrogen. This systematic error 
Table 7. Best fit parameters to RGS spectra in the $0.3-2.1 \mathrm{keV}$ range recorded in revolutions 536 (upper table) and 542 (lower table) using a multi-temperature plasma emission model built from the MEKAL code where the emission measures follow a power law in temperature, i.e. $E M(T)=N o r m \times\left(T / T_{\max }\right)^{\alpha}$. The oxygen and neon abundances were first tied to the abundance of the other elements (MODEL A) and were then left free to vary independently (MODEL B).

\begin{tabular}{cccc}
\hline \hline Rev. 536 & Parameter & MODEL A & MODEL B \\
\hline & $\alpha$ & $1.61 \pm 0.18$ & $1.32 \pm 0.17$ \\
& $T_{\max }(\mathrm{keV})$ & $2.5 \pm 0.4$ & $3.4 \pm 0.7$ \\
CEVMKL & Norm & $(7.2 \pm 0.8) \times 10^{-2}$ & $(5.8 \pm 0.8) \times 10^{-2}$ \\
& $\mathrm{O}$ & 0.24 & $0.24 \pm 0.05$ \\
& $\mathrm{Ne}$ & 0.24 & $0.9 \pm 0.2$ \\
& Other abundances & $0.24 \pm 0.04$ & $0.29 \pm 0.06$ \\
& $\chi^{2}$ & $0.95(521 / 549$ d.o.f. $)$ & $0.91(497 / 547$ d.o.f. $)$ \\
\hline \hline Rev. 542 & Parameter & MODEL A & MODEL B \\
\hline & $\alpha$ & $1.50 \pm 0.15$ & $1.32 \pm 0.14$ \\
CEVMKL & $T_{\max }(\mathrm{keV})$ & $4.0 \pm 0.7$ & $5.0 \pm 0.9$ \\
& Norm & $(9.6 \pm 0.9) \times 10^{-2}$ & $(8.5 \pm 0.8) \times 10^{-2}$ \\
& O & 0.29 & $0.28 \pm 0.05$ \\
& Other abundances & $0.29 \pm 0.05$ & $0.9 \pm 0.2$ \\
& $\chi^{2}$ & $0.97(735 / 758$ d.o.f. $)$ & $0.95(715 / 756$ d.o.f. $)$ \\
\hline
\end{tabular}
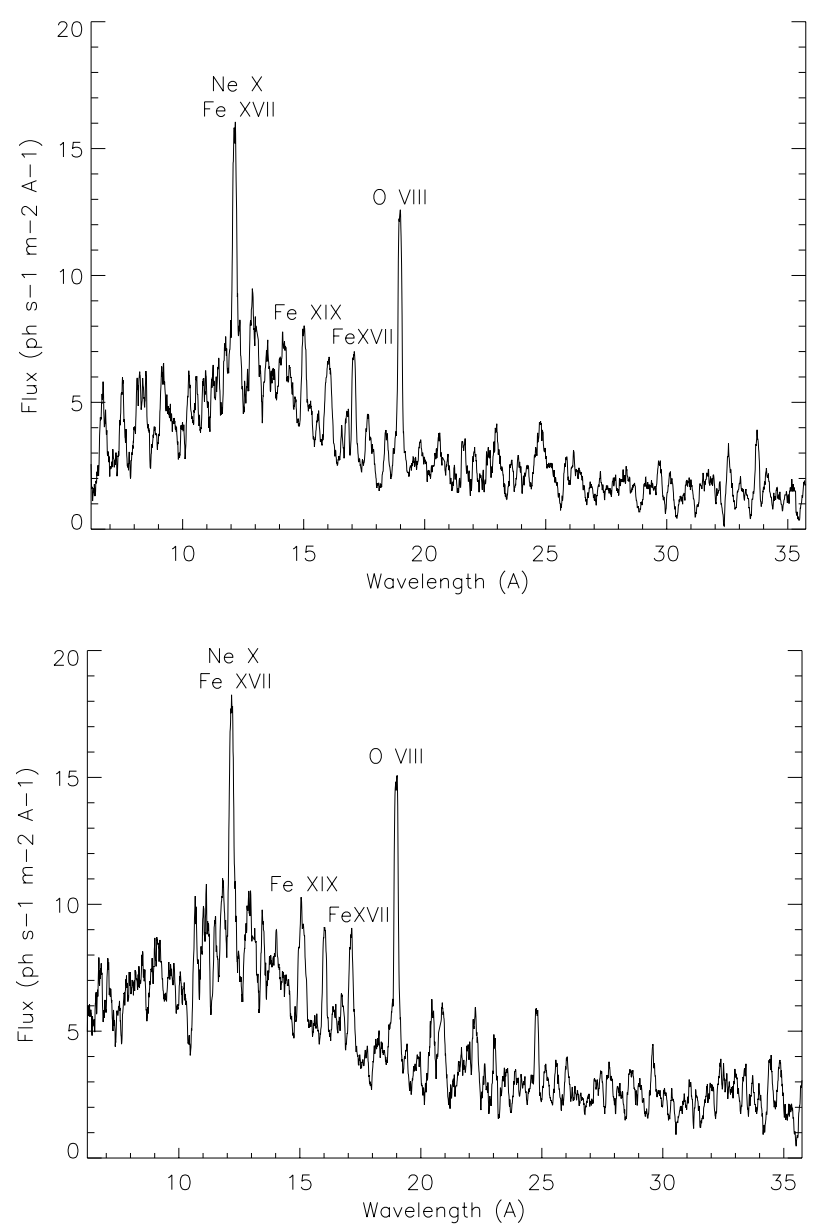

Fig. 4. Averaged first order spectra of RGS 1 and 2 obtained during revolutions 536 (top) and 542 (bottom). in the metal abundances relative to hydrogen is unknown but is expected to be small with respect to the abundance uncertainties stated in Table 6 . The fitting results suggest that neon abundance of the hot plasma component is higher than the oxygen abundance by a factor of 3 to 4 (see Tables 6 and 7). The Ne/O ratio found for HD 199178 is higher than in the solar photosphere. Such a Ne abundance enhancement is reminiscent of a similar anomaly observed in a subset of solar flares (Murphy et al. 1991; Schmelz 1993). Large Ne abundance enhancements are a common feature of active stellar coronae (Güdel et al. 2001; Huenmoerder et al. 2001) and an inverse FIP effect is observed in very active coronae (Brinkman et al. 2001; Drake et al. 2001) where the abundances (relative to oxygen) increase with increasing first ionization potential (FIP).

\section{Discussion}

The spectral fitting of the EPIC and RGS spectra of HD 199178 suggests a corona configuration with little contribution from quiet regions similar to the Sun. On the contrary the temperature $T \approx 8 \times 10^{6} \mathrm{~K}$ of the "cool" plasma component is reminiscent of solar type active regions, while the hot $\left(T>10^{7} \mathrm{~K}\right)$ components may be caused by disruptions of magnetic fields associated to a permanent flaring activity. The X-ray luminosity $\left(\approx(3.3-3.7) \times 10^{30} \mathrm{erg} \mathrm{s}^{-1}\right)$ of the "cool" component could be explained if $55-88 \%$ of the surface of HD 199178 is covered with bright solar like active regions (Vaiana \& Rosner 1978). Assuming that these active regions can be described by a simple static loop system consisting of similar loops of constant pressure $p\left(\mathrm{dyn} \mathrm{cm}^{-2}\right)$, temperature $T(\mathrm{~K})$ and cross section, the emission measure $E M\left(\mathrm{~cm}^{-3}\right)$ of the "cool" plasma can be expressed as:

$$
E M=G F \times\left(4 \pi R^{2}\right) \times\left(\frac{p}{2 k T}\right)^{2} \times L
$$



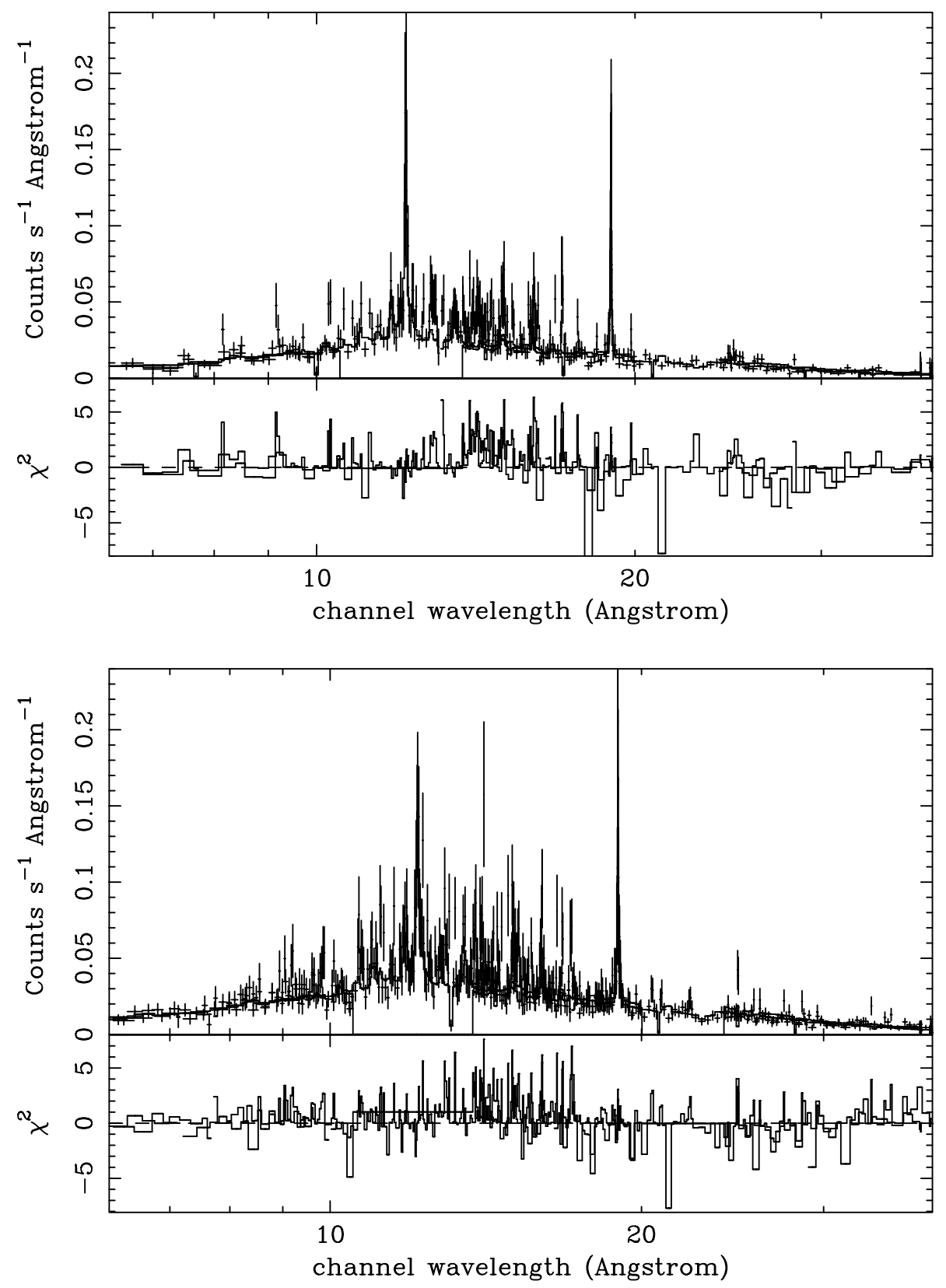

Fig. 5. Comparison of the RGS spectra of HD 199178 obtained during revolutions 536 (top) and 542 (bottom) with a best fit VMEKAL model with 3 temperature components (see Table 6, model B) in the 6-40 A range. The data (crosses) and spectral fit to the RGS spectra are shown in the upper panel. The $\chi^{2}$ contributions are given in the lower panel.

where $R$ is the stellar radius, $F$ is the filling factor and $L$ the loop half-length. $G$ is a geometry factor which includes effect of partial occultation of the corona by the star itself (i.e. $G$ varies from 0.5 to 1 for $L \ll R$ to $L \gg R$ ). Using the RTV relation $T=1400 \times(\mathrm{pL})^{1 / 3}($ Rosner et al. 1978) and $G=0.7$, a characteristic loop length scale is obtained (Mewe et al. 1982):

$L_{10}=7.4 \times F \times T_{7}^{4} \times E M_{52}^{-1} \times\left(R / R_{\odot}\right)^{2}$

where $L_{10}$ is the loop half length in units of $10^{10} \mathrm{~cm}, T_{7}$ is the coronal temperature in unit of $10^{7} \mathrm{~K}$, and $E M_{52}$ is the emission measure in units of $10^{52} \mathrm{~cm}^{-3}$. Inserting the observed temperature and emission measure of the cool plasma component (see Table 4 ) and $R=5 R_{\odot}$ (see Table 1 ), we find $L \approx 1.3 \times 10^{10} \mathrm{~cm}$ assuming a $\approx 70 \%$ filling factor for revolution 536 and 542 (see Table 8). Since it turns out that the loop length are much smaller than the pressure scale height $H$, the assumption of constant pressure in the loops is justified. Characteristic loop size and temperature on HD 199178 are respectively $1.3 \times 10^{10} \mathrm{~cm}$ and $8 \times 10^{6} \mathrm{~K}$. Solar corona observations by comparison show bright hot loops within active regions which reach maximum temperatures and electron densities above the neutral line of typically $(3-4) \times 10^{6} \mathrm{~K}$ and $10^{10} \mathrm{~cm}^{-3}$ (Vaiana et al. 1973). In addition to these hot loops, on-disk images of the Sun show that neighboring active regions are often connected into complexes of activity by large loop-like structures (Van Speybroek et al. 1970). Such interconnecting loops can be $>10^{10} \mathrm{~cm}$ long, i.e. 
Table 8. Physical parameters of HD 199178 coronal loops derived from the XMM-Newton observations conducted during revolution 536 and 542. The electron density is derived from Eq. (1), i.e $n_{\mathrm{e}}=2 \times$ $\sqrt{E M /\left(G F \times 4 \pi R^{2} \times L\right)}$.

\begin{tabular}{cccccc}
\hline \hline & $\begin{array}{c}E M \\
(\mathrm{~K})\end{array}$ & $\begin{array}{c}E M \\
\left(\mathrm{~cm}^{-3}\right)\end{array}$ & $\begin{array}{c}H \\
(\mathrm{~cm})\end{array}$ & $\begin{array}{c}L \\
(\mathrm{~cm})\end{array}$ & $\begin{array}{c}n_{\mathrm{e}} \\
\left(\mathrm{cm}^{-3}\right)\end{array}$ \\
\hline Rev. 536 & $8.1 \times 10^{6}$ & $43 \times 10^{52}$ & $56 \times 10^{10}$ & $1.4 \times 10^{10}$ & $1.3 \times 10^{10}$ \\
Rev. 542 & $7.9 \times 10^{6}$ & $35 \times 10^{52}$ & $55 \times 10^{10}$ & $1.3 \times 10^{10}$ & $1.2 \times 10^{10}$ \\
\hline
\end{tabular}

as long as the loop length estimate on HD 199178. However, they tend to be cooler than loops within solar active regions and therefore cooler than coronal loops on HD 199178.

The RTV scaling law neglects gravity, assumes a constant cross-section, uniform heating and constant pressure and a monotonic increase of temperature with height. The RTV scaling law is insentive to boundary conditions and depends only weekly on the nature of the heating mechanism (see e.g. Underwood et al. 1981). However, Schrijver et al. (1989) questioned the assumption of constant loop cross section. Schrijver (1987) noticed that coronal condensation over solar bipolar regions has a projected area roughly an order of magnitude larger than the area of the underlying photospheric plage, suggesting the ratio $\Gamma$ of the loop cross section at the apex to the cross section at the footpoint is approximately $\Gamma \approx 10$. Vesecky et al. (1979) made numerical calculations for loop with cross sections increasing with height in a way resembling a line dipole. They find that the scaling law is slightly modified by the change in the loop cross section with height. Schrijver et al. (1989) note that their numerical results can be conveniently approximated by a slightly modified scaling law, $T=1400 \Gamma^{-0.1} \times(p L)^{1 / 3}$. Using this modified law into Eq. (1), one finds that the constant $\Gamma$ enters Eq. (2) to the sixth power and could dramatically increase the loop half-length that we derived. Stern et al. (1986) point out that the expansion factor $\Gamma$ of a coronal loop is also the ratio of the magnetic strength at the loop base to that at the apex. Hence, one can argue that short low loops originating in the same active regions should have smaller $\Gamma$ than solar loops interconnecting different active regions. The length scale that we derived should be regarded only as an order of magnitude of the half length of hot loops on HD 199178, assuming a constant cross-section with height.

High temperature $\left(>10^{7} \mathrm{~K}\right)$ plasmas have been detected from the Sun and from non-solar coronae (van den Oord \& Mewe 1989; Tsuru et al. 1989; Antonucci \& Dodero 1995; Griffiths \& Jordan 1998; Sanz-Forcada et al. 2002). This aspect is much debated and still open, but it has been suggested that this hot component may be due to a continuous flaring activity (Güdel 1997; Drake et al. 2000). The surface of active stars is covered by active regions, and flares would be so frequent that their light curves overlap, canceling out any variability due to single events. This could explain the presence of hot material in the corona of HD 199178 even in the absence of obvious flares. There could be small scale flares not well identified in the light curves with moderate signal to noise ratio. The flatness of the HD 199178's light curve (see Fig. 1) could be explained by assuming that the heating of its corona results from a large number of small flares. Indeed, recent studies of the flare frequency in magnetically active stars as a function of the flare energy indicate power-law distributions that may be sufficient to explain all coronal radiative losses (Audard et al. 2000; Kashyap et al. 2002, Güdel et al. 2003). Within the continuous flaring hypothesis, the higher emission measure and luminosity contribution of the hot plasma component in revolution 542 would be related to a more intense flaring activity of HD 199178. On the other hand, the steady flux increase during revolution 542 could be interpreted as the gradual emergence of active regions at the limb of the star. Since active regions might not be homogeneously distributed on the surface of the star, it is difficult with the presented data to distinguish between a variable level of flaring activity and a rotational modulation of the X-ray emission by active regions.

HD 199178 was classified as an FK Comae type star due to its rapid rotation in regard to its spectral type and the apparent absence of a companion (Bopp \& Stencel 1981). The photometric period of HD 199178 is 3.327 days (Bopp et al. 1983; Jetsu et al. 1990), and a recent estimate of its equatorial velocity is $(76 \pm 7) \mathrm{km} \mathrm{s}^{-1}$ (see Table 1). Comparisons of $v \sin i$ measurements with theoretical models (Gondoin 2002, 2003a) suggests that HD 199178, which is evolving accross the Hertzsprung gap towards the bottom of the RGB, has not yet experienced rotational braking. At this stage of stellar evolution, $\alpha-\Omega$ dynamo mechanisms (Parker 1955) should operate with a maximum efficiency (Gondoin 1999). The X-ray luminosity of HD 199178 is indeed among the highest within a sample of single nearby F, G and K giants (Gondoin 2002, 2003a), thus supporting the above scenario. The X-ray to bolometric luminosity ratio of HD 199178, which is the highest among a sample of nearby giants, indicates that the high X-ray luminosity of HD 199178 rather results from a high surface density density of active regions related to its evolutionary position than from its large emitting surface. The coronal structure and evolutionary status of HD 199178 would thus be similar to that of FK Comae (Gondoin et al. 2002), V390 Aurigae (Gondoin 2003a) and HD 223460 (Gondoin 2003b). This justifies the classification of HD 199178 as an FK Comae-type star. These stars could be normal giants with A or B type progenitors on the main sequence that are evolving near the bottom of the red giant branch.

Acknowledgements. I thank my colleagues from the XMM-Newton Science Operation Center for their support in implementing the observations.

\section{References}

Allende Prieto, C., \& Lambert, D. L. 2001, ApJ, 556, L63

Anders, E., \& Grevesse, N. 1989, Geochim. Cosmochim. Acta, 53, 197

Antonucci, E., \& Dodero, M. A. 1995, ApJ, 438, 480

Arnaud, K., \& Dorman, B. 2001, XSPEC User's Guide for version 11.1, http://heasarc.gsfc.nasa.gov/docs/xanadu/ xspec/manual/manual.html

Audard, M., Güdel, M., Drake, J. J., et al. 2000, ApJ, 541, 396

Audard, M., Güdel, M., \& Mewe, R. 2001a, A\&A, 365, L318

Audard, M., Behar, E., Güdel, M., et al. 2001b, A\&A, 365, L329

Bopp, B. W., \& Stencel, R., E. 1981, ApJ, 247, L131 
Bopp, B. W., Africano, J. L., Stencel, R. E., et al. 1983, ApJ, 275, 691

Bowyer, S., Drake, J. J., \& Vennes, S. 2000, ARA\&A, 38, 231

Brinkman, A. C., Gunsing, C. J. T., Kaastra, J. S., et al. 2000, ApJ, 530,111

Brinkman, A. C., Behar, E., Güdel, M., et al. 2001, A\&A, 365, L324

Chen, B., Vergely, J. L., Valette, B., et al. 1998, A\&A, 336, 137

den Herder, J. W., Brinkman, A. C., Kahn, S. M., et al. 2001, A\&A, $365, \mathrm{~L} 7$

de Medeiros, J. R., \& Mayor, M. 1995, A\&A, 302, 745

Dempsey, R. C., Bopp, B. W., Strassmeier, K. G., et al. 1992, ApJ, 392, 187

Dickey, J. M., \& Lockman, F. J. 1990, ARA\&A, 28, 215

Doschek, G. A., \& Cowan, R. D. 1984, ApJS, 56, 67

Drake, J. J., Peres, G., Orlando, S., et al. 2000, ApJ, 545, 1074

Drake, N. A., Brickhouse, N. S., Kashyap, V., et al. 2001, ApJ, 548, L81

Dupree, A. K., Brickhouse, N. S., Doschek, G. A., et al. 1993, ApJ, 418, L41

Durney, B. R., \& Latour, J. 1978, Geophysical and Astrophysical Fluid Dynamics, 9, 241

Durney, B. R., De Young, D. S., \& Roxburgh, I. W. 1993, Sol. Phys., 145, 207

Ehle, M., Breitfellner, M., Dahlem, M., et al. 2001, The XMM-Newton Users' Handbook, http://xmm.vilspa.esa.es/user/A02/uhb/xmm_uhb.html

Endal, A. S., \& Sofia, S. 1979, ApJ, 232, 531

ESA 1997, The Hipparcos Catalogue, ESA SP-1200

Fekel, F. C. 1997, PASP, 109, 514

Flower, P. J. 1996, ApJ, 469, 335

Gabriel, A. H., \& Jordan, C. 1969, MNRAS, 145, 241

Gilliland, R. L. 1985, ApJ, 299, 286

Golub, L., \& Pasachoff, J. M. 1997, in The Solar Corona (Cambridge, UK: Cambridge University Press)

Gondoin, P. 1999, A\&A, 352, 217

Gondoin, P., Aschenbach, B., Erd, C., et al. 2000, SPIE Proc., 4140, 1 Gondoin, P., Erd, C., \& Lumb., D. 2002, A\&A, 383, 919

Gondoin, P. 2003a, A\&A, 404, 355

Gondoin, P. 2003b, A\&A, 409, 263

Gray, D. F. 1989, ApJ, 347, 1021

Gray, D. F., \& Endal, A. S. 1982, ApJ, 254, 162

Griffiths, N. W., \& Jordan, C. 1998, ApJ, 497, 1998

Güdel, M. 1997, ApJ, 480, L121

Güdel, M., Guinan, E. F., Mewe, R., et al. 1997a, ApJ, 479, 416

Güdel, M., Guinan, E. F., \& Skinner, S. L. 1997b, ApJ, 483, 947

Güdel, M., Audard, M., Briggs, K., et al. 2001a, A\&A, 365, L336

Güdel, M., Audard, M., Magee, H., et al. 2001b, A\&A, 365, L344

Hackmann, T., Jetsu, L., \& Tuominen, I. 1999, A\&A, 374, 171

Herbig, G. H. 1958, ApJ, 128, 295

House, L. L. 1969, ApJS, 18, 2

Huenemoerder, D. P. 1986, AJ, 92, 673

Huenemoerder, D. P., Canizares, C. R., \& Schulz, N. S. 2001, ApJ, 559,1135

Hünsch, M., Schmitt, J. H. M. M., \& Voges, W. 1998, A\&AS, 127, 251

Jansen, F., Lumb, D., Altieri, B., et al. 2001, A\&A, 365, L1

Jetsu, L., Huovelin, J., Tuominen, I., et al. 1990, A\&A, 236, 423

Jetsu, L., Pelt, J., \& Tuominen, I. 1999, A\&A, 351, 212

Kashyap, V. L., Drake, J. J., Güdel, M., et al. 2002, ApJ, 580, 1118

Kucera, T. A., Dennis, B. R., Schwartz, R. A., et al. 1997, ApJ, 475, 738

Kurucz, R. L. 1991, in Stellar Atmospheres: Beyond Classical Models, NATO ASI Series C, vol. 341

Lemen, J. R., Mewe, R., Schrijver, C. J., et al. 1989, ApJ, 341, 474
Leonard, P. J. T., \& Livio, M. 1995, ApJ, 447, L121

Lestrade, J. F., Prestom, R. A., Jones, D. L., et al. 1999, A\&A, 344, 1014

Liedahl, D. A., Osterheld, A. L., \& Goldstein, W. H. 1995, ApJ, 438, 115

Maggio, A., Vaiana, G. S., Haisch, B. M., et al. 1990, ApJ, 348, 253

Makishima, K. 1986, in The Physics of Accretion onto Compact Objects, p. 250, ed. K. O. Mason, M. G. Watson, \& N. E. White (Berlin: Springer Verlag)

Mewe, R., Gronenschild, E. H. B. M., Heise, J., et al. 1982, ApJ, 260, 233

Mewe, R., Gronenschild, E. H. B., \& van den Oord, G. H. J. 1985, A\&A, 62, 197

Mewe, R., Kaastra, J. S., White, S. M., et al. 1996, A\&A, 315, 170

Mewe, R., Raassen, A. J. J., Drake, J. J., et al. 2001, A\&A, 368, 888

Murphy, R. J., Ramaty, R., Reames, D. V., et al. 1991, ApJ, 371, 793

Osten, R. A., Ayres, T. R., Brown, A., et al. 2003, ApJ, 582, 1073

Parker, E. N. 1955, ApJ, 122, 293

Pizzolato, N., Maggio, A., \& Sciortino, S. 2000, A\&A, 361, 614

Pradhan, A. K. 1982, ApJ, 263, 477

Raymond, J. C., \& Smith, B. W. 1977, ApJS, 35, 419

Reale, F., Peres, G., \& Orlando, S. 2001, ApJ, 557, 906

Rogers, F. J., \& Iglesias, C. A. 1992, ApJS, 79, 507

Rosner, R., Tucker, W. H., \& Vaiana, G. S. 1978, ApJ, 220, 643

Rosner, R., Musielak, Z. E., Cattaneo, F., et al. 1995, ApJ, L442, L25

Rutten, R. G. M., \& Pylyser, E. 1988, A\&A, 191, 227

SAS 2001, XMM-Newton Science Analysis System, Reference Documentation, http://xmm.vilspa.esa.es/user/ sas_top.top.html

Sanz-Forcada, J., Brickhouse, N. S., \& Dupree, A. K. 2002, ApJ, 570, 799

Schaller, G., Schaerer, D., Meynet, G., et al. 1992, A\&AS, 96, 269

Schmelz, J. T. 1993, ApJ, 408, 373

Schmitt, J. H. M. M., Collura, A., Sciortino, S., et al. 1990, ApJ, 365, 704

Schmitt, J. H. M. M., Drake, J. J., \& Stern, R. A. 1996, ApJ, L465, 51

Schmitt, J. H. M. M., \& Ness, J. U. 2002, A\&A, 388, L13

Schrijver, C. J. 1987, A\&A, 180, 241

Schrijver, C. J., Lemen, J. R., \& Mewe, R. 1989, ApJ, 341, 484

Schrijver, C. J., van den Oord, G. H. J., Mewe, R., et al. 1995, A\&A, 302,438

Sills, A., Lombardi, J. C., Bailyn, C. D., et al. 1997, ApJ, 487, 290

Simon, T., \& Drake, S. A. 1989, ApJ, 346, 303

Strassmeier, K. G., Lupinek, S., Dempsey, R. C., et al. 1999, A\&A, 347,212

Strüder, L., Briel, U., Dennerl, K., et al. 2001, A\&A, 365, L18

Tsuru, T., Makishima, K., Ohashi, T., et al. 1989, PASJ, 41, 679

Turner, M. J. L. T., Abbey, A., Arnaud, M., et al. 2001, A\&A, 365, L27

Underwood, J. H., Antiochos, S. K., \& Vesecky, J. F. 1981, in Solar Phenomena in Stars and Stellar Systems, ed. R. M. Bonnet, \& A. K. Dupree (Dordrecht: Reidel), 227

Vaiana, G. S., Davis, J. M., Giacconi, R., et al. 1973, ApJ, 185, 47

Vaiana, G. S., \& Rosner, R. 1978, ARA\&A, 16, 393

van den Oordt, G. H. J., \& Mewe, R. 1989, A\&A, 213, 245

Van Speybroeck, L. P., Krieger, A. S., \& Vaiana, G. S. 1970, Nature, 227,818

Vesecky, J. F., Antiochos, S. K., \& Underwood, J. H. 1979, ApJ, 233, 987

Vogt, S. S. 1988, in The Impact of Very High S/N Spectroscopy on Stellar Physics, ed. G. Cayrel de Strobel, \& M. Spite (Dordrecht: Kluwer Academic Publishers), IAU Symp., 132, 253

Walter, F. M., \& Basri, G. S. 1982, ApJ, 260, 735

Walter, F. M., Neff, J. E., Bopp, B. W., et al. 1984, in Cool Stars, Stellar Systems, and the Sun, ed. S. L. Baliunas, \& L. Hartmann (Springer-Verlag), Vol. 193, p. 279 